\title{
OpenLitterMap.com - Open Data on Plastic Pollution with Blockchain Rewards (Littercoin)
}

\author{
Seán Lynch®
}

\begin{abstract}
OpenLitterMap rewards users with Littercoin for producing open data on litter. Open data on the geospatial characteristics of litter provide means of invoking and evaluating responses to plastic pollution. OpenLitterMap currently works as a web app on all devices with native mobile apps in development. The stack includes the integration of the Laravel PHP Framework on the backend; Vue for frontend reactivity; NativeScript-Vue for mobile apps; Bulma for CSS; Leaflet for web-mapping; Turf.js for geospatial analysis; the Ethereum Blockchain for tokenization; Stripe; ChartJS; AWS; and more. Anywhere from a single cigarette butt to the contents of an entire beach or street clean can be logged in a single geotagged photo. Alternatively, a simple index may be used if litter is incalculable. The open data includes an increasing 100+ pre-defined types of litter; 20+ corporate brands; verification status; coordinates; timestamp; phone model; the latest OpenStreetMap address at each location; and the litter presence as a Boolean. To date, 100\% of all submitted data ( 8200 photos, 28,000 litter from over 150 contributors) has been manually verified which is being used to develop machine learning algorithms.
\end{abstract}

Keywords: OpenLitterMap, Littercoin, Plastic pollution, Open data, Blockchain, Machine learning

\section{Introduction}

Human activities on the environment have been so severe that we (humans) have literally defined the "Anthropocene" [27]. The Anthropocene is the latest epoch in the geologic history of the Earth, characterised exclusively by humans' impact on the environment. Although there are many factors involved (e.g. climate change, deforestation, etc.), perhaps nothing characterises the Anthropocene more than plastic pollution, which was first recognized to have a global distribution in the marine environment as early as 1975 [21]. In cities, nicotine has become a recognized pollutant of urban waters because of the trillions of plastic-tipped cigarette butts dropped around the world [9]. Since the beginning of commercial plastic production in the 1950s, the annual global production of plastic rose to 30-million tonnes by 1988 and to $280-299$ million tonnes by 2014 $[19,26]$. In this time we have embedded plastic, potentially permanently, right across our only habitable planet. Recent research from The Ocean Cleanup ${ }^{1}$ in the Pacific

Correspondence: info@openlittermap.com

OpenLitterMap, Cork, Ireland
Ocean suggests that the "Great Pacific Garbage Patch", the largest accumulation of plastic in the oceans, spans an area of about 1.6 million $\mathrm{km}^{2}$ weighing about $80,000 \mathrm{t}$, which is 4 and 16 times larger than previous estimates and is now said to be rapidly accumulating more plastic [16]. On land, litter, or plastic pollution, costs taxpayers millions in many countries every year for cleanup intervention and management as well as potentially infinitely more in biodiversity and ecosystem service loss. The UNEP [26] reports that globally, 22$43 \%$ of plastic is disposed of in landfills, which have a limited capacity thus increasing the costs of waste management and waste security over time. The World Bank estimated that this quantity of waste and its related cost will likely double from 2012 to 2025 [11]. Despite most plastic originating on land, plastic pollution is now one of the most serious threats for healthy marine ecosystem functioning $[1,14,20,25,26]$. Traditionally, because of spatial and temporal restrictions, it was only possible for scientists to make inferences on the spatial distribution of litter based on small samples of data. Today, hundreds of millions of 
people are equipped with incredibly accurate geospatial data collectors, which can be used to map and crowdsource geospatial data not just on plastic pollution, but to some extent, the litter caused by the products and economic activity of a handful of global corporations. Depending on the nature of projects and the data collection methods, this crowdsourcing of data is commonly referred to as Citizen Science [13] and Volunteered Geographic Information (VGI). Crowdsourced data is a paradigmatic shift in how knowledge, particularly that of a geospatial nature, is created and shared throughout society [7]. The need for geospatial information to mitigate and raise awareness about our plastic footprint is urgent. In 2010 alone, it has been estimated that between 8 and 12 million tonnes of plastic and other types of waste entered the oceans. If current trends continue, this figure is expected to continue to increase exponentially potentially reaching $\sim 70$ million tonnes by 2025 [14]. Some authors have estimated that $90 \%$ of plastic waste entering the ocean is from just 10 rivers. However, the same authors acknowledge there are large uncertainties with these estimates because of a lack of data [23]. The Ocean Cleanup estimate the annual global input of plastic into the ocean by rivers is between 1.15-2.41 million tonnes, acknowledging that the top 20 polluting rivers are primarily located in Asia, which the authors account for up to $67 \%$ of the global input [17]. However, these studies focus largely on population and waste management metrics and because of a lack of geospatial data on plastic pollution, these approaches may be largely unable to appropriately characterize contemporary anthropogenic litter [12].

Many efforts have been made at involving citizens as data collectors. OpenStreetMap (OSM) is a landmark project that enables citizens to collect and share geospatial data. In 2008, Haklay \& Weber anticipated that OSM would provide a good example of the social and technical aspects of user-generated content communities [10]. In fact, their opinion turned out correct as several research and operational projects have been employing OSM data and its concept in data collection (e.g. OpenLitterMap, OpenTreeMap, OpenCycleMap, etc.). More recent studies present tools and methods based on OSM to enrich the database with detailed information about the accessibility of roads and buildings [2, 18, 22]. Please note that these studies are only a few examples of a wide variety of research carried out in several application domains such as emergency response [8], urban studies [3] as well as noise pollution [15]. ${ }^{2}$ In terms of plastic pollution, NGO Coastwatch ${ }^{3}$ lead by Karin Dubsky of Trinity College Dublin has been pioneering citizen science plastic bottle mapping on Irish beaches since at least 1991, and coastal biodiversity monitoring more generally since the 1970s. During their annual survey in September 2017, over 8800 plastic bottles were found at 583 sections of the Irish coastline $(3.6 \text { per } 100 \mathrm{~m})^{4}$ which has to be taken into consideration following the large increase

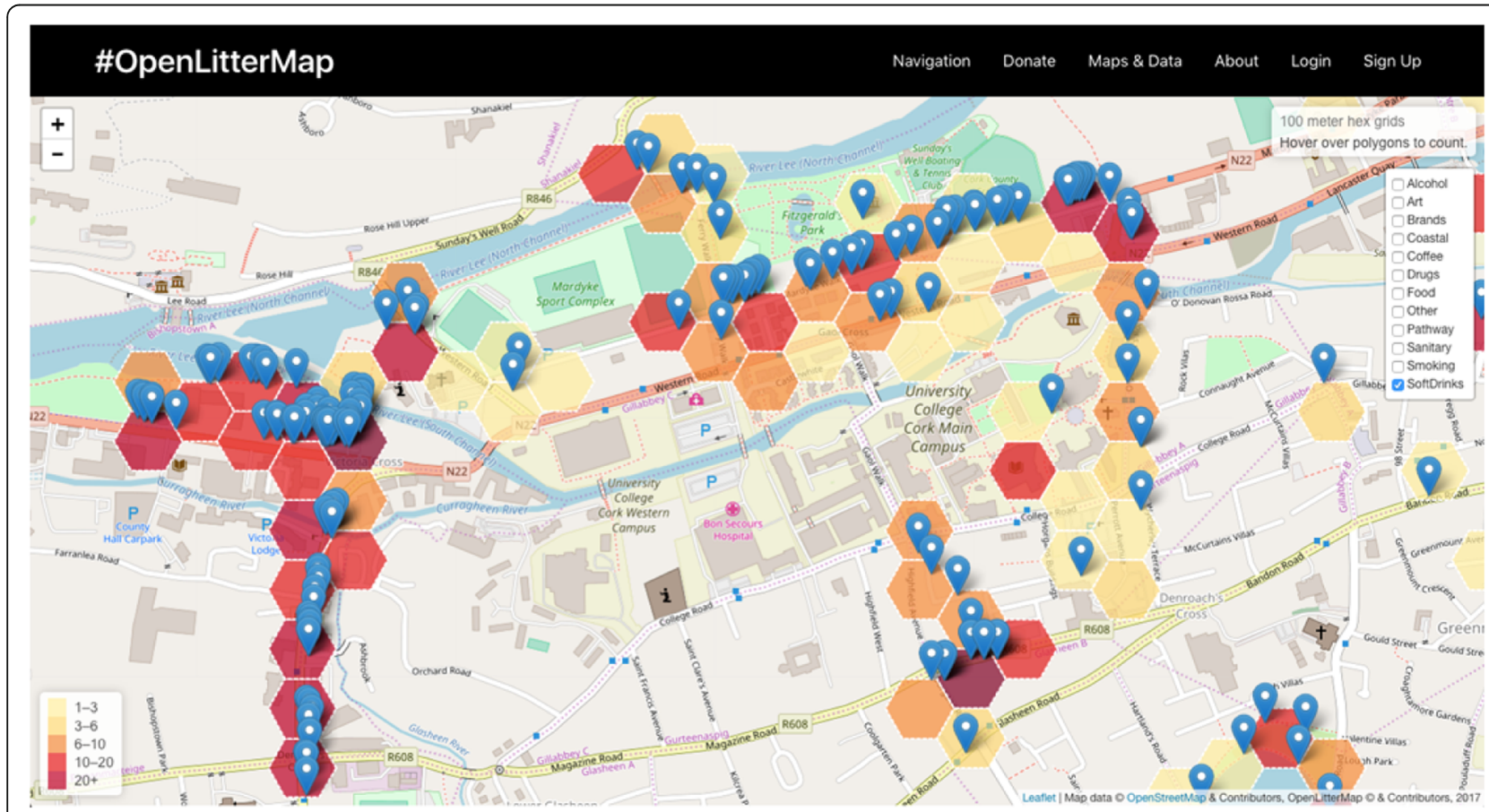

Fig. 1 OpenLitterMap Data on Plastic Pollution around UCC, Cork, Ireland https://openlittermap.com/maps/lreland/County Cork/Cork/map 
in the number of beach cleans over the years who focus more on removal than statistics. One authoritative example of geospatial information reporting is the "See it, Say it" app from the Environmental Protection Agency in Ireland ${ }^{5}$ that can be used to report incidents of illegal dumping or litter blackspots with a Local Authority. Local authorities have advanced mapping capabilities and are usually associated with managing waste and pollution, however Local Authorities are not typically interested in mapping the widespread abundance and distribution of litter caused by the economic activity of global corporations in a detailed, open and accessible manner. To fill this gap, several startup, research and institutional efforts have emerged with a specific focus on crowdsourcing geospatial data on litter. One example, Litterati, ${ }^{6}$ claim to have crowdsourced over 1 million Instagram-based hashtags of litter which are available to explore on a global map. However, none of this data is available for download and it is not clear if and how irrelevant hashtags are filtered out. Userdefined hashtags have become popular as a means to crowdsource data as existing and established services such as Instagram and Twitter enable people to share geographic information for free. While hashtagging opens up a wide possibility of feature classifications, in general, user-defined hashtags are error prone, arbitrary, inconsistent, computationally expensive, lack quantification, and ultimately limit data quality and utility. In terms of accessible crowdsourced and scientific data that is available for download, the largest include Litterbase $^{7}$ from the Alfred Wegner Institute (AWI) of Germany who have collated data from 792 publications on marine litter [24]; Marine Debris Tracker $^{8}$ from the United States' National Oceanographic and Atmospheric Administration (NOAA) who have crowdsourced over 1. 2 million tags of marine debris; Project AWARE $^{9}$ (0.96 million pieces from PADI); and Clean Swell ${ }^{10}$ (37 million lbs. of trash removed and recorded) from the Ocean Conservancy. Many projects have focused significantly more on marine litter than its' pre-marine terrestrial and corporate characteristics. If we are to improve our relationship with and ability to respond to plastic pollution, significantly more emphasis needs to be put on crowdsourcing and producing detailed and accessible knowledge on the geospatial characteristics of plastic pollution in cities, along riverbanks, across beaches and coastlines, around schools and hospitals, national parks, protected areas and anywhere else where plastic pollution can be found. To advance our ability at understanding and tackling plastic pollution, all data at OpenLitterMap goes through a verification process before being made completely free and open for anyone to download (Fig. 1; Open Database License); All data is pre-defined based on independent research; OpenLitterMap builds its list of locations dynamically while maintaining native languages and alphabets where possible (a process that is redeemable in Littercoin); All verified data is automatically and dynamically analyzed by space, time, location, and behaviour everywhere by design; OpenLitterMap includes litter presence to determine if litter has been taken away or if it is still remaining; And OpenLitterMap is the first to reward contributors with an original blockchain token Littercoin as a reward for the production of open geospatial data. Not only can we now crowdsource and produce open geospatial data on litter at a global scale but with a potential monetary or utility incentive that is being made possible through blockchain technology (See: Section 2.1.3 Littercoin), we might be able to incentivize and facilitate the rapid production of geospatial data at a global scale at an unprecedented rate never seen before and get a good picture of many parts of the worlds litter in as little as 1 or $2 \mathrm{~h}$. However, if we are to achieve this level of open data at a global scale one basic question needs to be asked. Who will cover the costs of hosting open data and financing the development of OpenLitterMap? Is open data sustainable or is "ClosedLitterMap" a more realistic paradigm? Can we use this data to enforce polluter pays principles to generate necessary revenue? To achieve some degree of sustainability, a non-profit, the OpenLitterMap Foundation is being established. This legal entity will be able to accept monthly crowdfunding contributions. Just like the OpenStreetMap Foundation, this entity will be able to improve and democratize the development of the OpenLitterMap ecosystem by giving members a voice to guide and promote future developments. ${ }^{11}$ If the reader would like to see progress they are encouraged to participate in crowdfunding the development of OpenLitterMap with small monthly micro-financial contributions at openlittermap.com/signup with as little as $€ 5$ a month. Without support, the future of OpenLitterMap is uncertain [16].

\section{Implementation}

OpenLitterMap.com (Registered 27th July 2015, Launched 15th April 2017) rewards users with Littercoin for doing the work of collecting, mapping, processing and producing open geospatial data on an increasing variety of pre-defined types of litter including corporate brands anywhere. This process is currently accomplished through a web-app, which is built mobile first and available on all devices at openlittermap.com. To ensure data quality, all data goes through a manual verification process, which is being done by a small team of trained volunteers. This verified data is being used to develop machine-learning algorithms that will make manual processing and verification easier. However, without an 
immediate and continued source of funding the future of OpenLitterMap is uncertain.

\section{Technologies used \\ Backend - PHP}

Laravel 5.4 (https://laravel.com) is used as a backend framework as it provides a robust and flexible foundation for developing a geospatially-enabled API.

\section{Frontend - JavaScript}

To render dynamic components reactively in the browser, such as Pie charts and Time-series, Vue.js (https://vuejs.org) is used in combination with ChartJs (https://chartjs.org). For maps, Leaflet (https://leafletjs. $\mathrm{com} /$ ) is used to display OpenLitterMap categorized point data, which is layered over OpenStreetMap (openstreetmap.org) tiles. An auto-clustering plugin for Leaflet is employed for the global map, and Turf (htttps://turfjs.org) is layered between points and tiles to achieve a dynamic weighted hexagonal spatial grid at each City layer.

\section{Littercoin}

The creators of open data at OpenLitterMap are rewarded with Littercoin, a token on the Ethereum blockchain as a reward for proof of their work. This is analogous to the proof of work mining protocol on blockchains such as Bitcoin and Ethereum. However, because the production of Littercoin is not as financially or technically restrictive as producing Bitcoin or Ether, Littercoin (although facilitated by Ethereum) makes the "mining" (i.e. reward) of a token even more accessible. Although a monetary value or general utility has yet to be defined, users may begin collecting and trading these tokens with others who value their creation. As cryptocurrencies are typically permissionless, the possibilities for Littercoin are endless. For example, a business that appreciates the work being done by data producers could offer a reward (eg. a coffee or a discount or a sample) in exchange for some Littercoin. Such businesses could then use an accumulation of Littercoin as a costeffective marketing strategy to attract more customers and showcase their appreciation of the work being done by the OpenLitterMap community. As Littercoin has the potential to incentivize the production of data that can increase the cost-effectiveness of existing public services, local governments could use it as a tax incentive, or to offer proactive healthy initiatives to citizens such as access to off-peak swimming pool hours, or for giving members of disadvanteged communites increased access to other goods and services, such as discounted fresh fruit and vegetables. Currently, 1 Littercoin is rewarded for every 100 images sent for successful verification in a row. This is intended to incentivize people to submit data correctly. To incentivize early adoption, OpenLitterMap will reward users for being the first to upload from a new location. Simply by uploading to OpenLitterMap, users dynamically populate the list of available locations in the database. Users receive 100 Littercoin for the being the first to upload from a Country, 50 Littercoin for the creation of a State, and 25 Littercoin for each City (Note, that these terms are used as a generalization for each of the 3 layers which are based on unrecogized OpenStreetMap values during the upload process). Although there are no restrictions as to how many locations a user can create, there are only 10 million Littercoin in the current v1.0 model, a number which was chosen arbitrarily. Due to technical smart contract limitations, Littercoin rewards are currently being distributed manually. While it doesn't cost anything to receive tokens, it costs a fraction of eth to send tokens ( €0.10-20c). To claim allowances, the authenticated user must visit openlittermap.com/settings/littercoin and enter an Ethereum public wallet ID or follow the instructions to create one. A more detailed whitepaper on Littercoin will follow this article about the future vision for this "GeoBlockchain" ecosystem and outline the business details about how we might be able to create a monetary incentive to reward people for producing open data on plastic pollution. Such a reward may have the potential to incentivize the rapid crowdsourcing of geospatial information at a global scale never seen before.

\section{The OpenLitterMap Foundation}

OpenLitterMap is currently entirely dependent on (and lacking in) crowdfunding contributions. Although open data has considerable socio-economic, environmental and governance benefits, it is does not provide an immediate or reliable source of revenue. Without an immediate and continued source of funding, the future of OpenLitterMap is not only uncertain, but bleak. Finances to map and produce open data on the pollution being caused largely by the products of a small number of global corporations have not been identified. To accept donations and give members a voice in the future of the OpenLitterMap ecosystem, a non-profit, the OpenLitterMap Foundation is being established. Several Ambassadors have joined the pre-liminary team from Ireland, Australia, the U.K., and the U.S.A. who are interested in promoting OpenLitterMap locally and we hope to have our first online AGM and elect the first committee in 2018. Following the development of more advanced and potentially commercial features we aim to transfer the cost away from data producers to sources of pollution (eg. corporations) who need to improve their image and social responsibility. Similarly, data consumers (eg. Local Authorities) need advanced analytics 
and near-real time insights. However, because litter mapping is a relatively new field of Geographical Information Science, neither of these institutions have the structures in place to enable the development of OpenLitterMap. In lieu of a grant or philanthropy, microfinancial monthly crowdfunding contributions averaging $€ 5$ from just $~ 1000$ individuals worldwide who believe in and would like to see OpenLitterMap survive and be developed further offer a means of achieving growth and sustainability. To facilitate monthly crowdfunding donations, stripe.com provides a secure and established way of processing euros, pounds and dollars over an encrypted network. Subscriptions can be created on registration or cancelled and reactivated via openlittermap.com/settings/payments in a few simple clicks. Donations can also be made via Stripe, or by using trustless decentralized stateless payment infrastructures such as Bitcoin and Ethereum at openittermap.com/donate. By crowdfunding the development of OpenLitterMap, users can enjoy the updates and will be able to participate in guiding future developments. This could also be conducted using blockchain technology through a DAO (Decentralized Autonomous Organisation), which would allow members to vote on proposals with Littercoin or some other token. Interested parties should contact the author.

\section{Databases}

Data for OpenLitterMap is stored and made available through the integration of MySQL and NoSQL (Redis).

\section{Hosting and SSL}

OpenLitterMap is hosted by AWS (Amazon Web Services) who generously awarded OpenLitterMap with $\$ 1000$ start-up credits as part of their Activate program. Post mid-January 2018 images are hosted on an Amazon S3 bucket. During the switch to S3, images uploaded prior to this were accidentally deleted which is why some images have been replaced with a green checkmark. An nginx server was provisioned with Laravel Forge (https://forge.laravel.com), which also provides free SSL certificates via Let's Encrypt.

\section{CSS and animation}

OpenLitterMap is styled with the CSS Framework Bulma (https://bulma.io) and uses WOW.js for animation (https://mynameismatthieu.com/WOW/).

\section{Dropzone}

Dropzone (dropzonejs.com) is used to enable users to share geotagged images. Only geotagged images can be submitted and the user is restricted from uploading the same image more than once. During the upload process, GPS tags are reversed geocoded with OSM to get the address at each location. Unrecognized address values are used to dynamically populate the list of available locations, which is redeemable in Littercoin. All OSM address data is included in the downloads.

\section{Mobile apps}

Native mobile apps for iOS and Android are being developed with the new NativeScript-Vue (CLI v0.2) framework, ${ }^{12}$ which OpenLitterMap is proudly supporting the development of. ${ }^{13}$ However if necessary libraries are not available we may temporarily switch to ReactNative until NativeScript-Vue is more developed.

\section{Maps}

OpenLitterMap provides a global auto-clustering spider map at openlittermap.com/global. Furthermore, a $100 \mathrm{~m}^{2}$ weighted hexagonal spatial grid is applied at each of the City layers where more than 1 photo exists. The user may visit different locations by navigating through the list of available locations or by directly manipulating the URL (e.g. openlittermap.com/maps/UK/England/London/map). Each cell is weighted spatially by the number of items of litter in each space not the number of photos, as one photo can have many litter items associated with it. Using the options panel available at each City layer (replace "/map" with "/options"), data can be filtered by the day and the size of the hex can be changed from a range of $1-500 \mathrm{~m}^{2}$ simply by moving the sliders available on the options panel or by directly manipulating the updated URL.

\section{Methodology Litter classification}

An increasing 100+ types of litter have been pre-defined by OpenLitterMap, which are mapped by several behavioral categories of related-waste, coastal litter, litter art and global corporations. These were selected based on independent research and continue to improve with feedback and the submission of previously unidentified items.

Smoking Cigarette/Butts, Lighters, Cigarette Box, Tobacco Pouch, Rolling Papers, Plastic Packaging, Filters, Filter box, Smoking-Other; (9);

Alcohol Glass Bottles (beer), Glass Bottles (Spirits), Glass Bottles (Wine), Beer Cans, Broken Glass, Beer Bottle Tops, Paper Packaging, Plastic Packaging, Alcohol-Other; (10);

Coffee Coffee Cups, Coffee Lids, Coffee-Other; (3);

Food Sweet Wrappers, Paper/Card Packaging, Plastic Packaging, Plastic Cutlery, Crisp/Chip Packet Small, 
Crisp/Chip Packet Large, Styrofoam Plate, Napkins, Sauce Packet, Glass Jar, Glass Jar Lid, Food-Other; (13);

Soft-drinks Plastic Water Bottle, Plastic Fizzy Drink Bottle, Tin can, Bottle tops, Bottle label, Sports Drink Bottle, Straws, Plastic Cups, Plastic Cup Tops, Milk Bottle, Milk Carton, Paper Cups, Juice Cartons, Juice Bottles, Juice Packet, Ice Tea Bottles, Ice Tea Can, Energy Can, Soft Drink (other); (19);

Drugs Needles; Citric Acid Packets; Alcohol Wipes; Needle Tops; Needle Packaging; Sterile Water Bottles; Spoons; Needle Bins; Tinfoil; Syringe Barrels; Unopened "Full Package"; Baggie; Crack Pipes; Other; (13); ${ }^{14}$

Sanitary Condoms, Nappies, Menstrual, Deodorant, Ear Swabs, Tooth Pick, Tooth Brush, Sanitary (other); (8);

Other Dogshit, Unidentified Plastic, Large/Random Dump, Metal Object, Plastic Bags, Election Posters, For Sale Posters, Books, Magazines, Paper, Stationary, Washing-up Bottle, Hair tie, Ear Plugs, Other (other); (17);

Coastal Microplastics, Mediumplastics, Macroplastics, Rope small, Rope medium, Rope large, Fishing Gear/ Nets, Buoys, Degraded Plastic Bottle, Degraded Plastic Bag, Degraded Drinking Straws, Degraded Lighters, Balloons, Lego, Shortgun Cartridges, Coastal (other); (15);

Pathway ${ }^{15}$ Gutter, Gutter (long), Kerb hole (small), Kerb hole (large), Pathway (other); (5);

\section{Art (introduced 5th Oct 2017) Item; (1);}

Brands (introduced 16th February 2018) Adidas, Amazon, Apple, Budweiser, Coca-Cola, Colgate, Corona, Frito-Lay, Gillette, Heineken, Kellogs, Lego, Loreal, Nescafe, Nestle, Marlboro, McDonalds, Nike, Pepsi, Rebull, Samsung, Subway, Starbucks, Tayto (24). ${ }^{16}$

\section{Producing open data for verification}

On login, users are redirected to the submit page (openlittermap.com/submit). On this page, users can click on the large dropzone box to gain access to their photo gallery or simply drop geotagged photos on the box to upload them. To process data and tag litter to images, the user must visit openlittermap. $\mathrm{com} /$ profile. This is linked twice. Once towards the bottom of the submit page and again towards the bottom of the drop-down navigation menu which is available from the upper-right hand corner. On the profile page, the user must manually attribute how much litter appears in each photo. Anywhere from a single cigarette butt to the contents of an entire beach or street clean can be logged in a single photo. If too many items are present and the user is unable to process all of the items individually, OpenLitterMap gives the option for a "Large/Random Dump" in the "Other" category that can be used to rank an area from a simple index of 1-100. Once the litter in the image has been attributed, it must be submitted for verification.

\section{Verification}

All OpenLitterMap is being manually verified to achieve a trusted database. This is currently achieved through a custom admin interface that can achieve successful verification of correctly categorized images at a rate of about 1 per second. Falsely attributed images are either rejected and sent back to the user for re-classification or corrected and accepted. Recently, several Ambassadors have volunteered to help with the verification process. Each Ambassador selected for this process has been correctly submitting data diligently for some time, and was chosen carefully as they demonstrated integrity and received some training to ensure best practices. Upon verification, all verified data is dynamically analyzed by space, time, location, behaviour and corporation, and is made free and open by design. Plans are in place to manage an expected backlog of unverified photos, such as by releasing unverified or semi-verified data. Adminapproved verification is considered "Stage 2" level of verification. Users can also vote true or false on each other's images to achieve Stage 1 level of verification. However at this time there is rarely a backlog of images that are available for Stage 1 level of verification. Upon either stage of verification, all maps, charts and downloads will automatically update. If an image receives a certain number of false verifications it will be flagged for admin inspection and will not be included in the available data. Currently, $100 \%$ of submitted images have been verified to Stage 2 level of verification and can be considered admin-approved manually verified free and open citizen science data.

\section{Adding new locations}

Users add new locations to OpenLitterMap simply by being the first to upload a geotagged photo of litter from that location. OpenLitterMap reverse geocodes each set of GPS coordinates to get the latest OpenStreetMap address at each location and uses this information to populate its list of locations dynamically. To incentivize early adoption, this process is redeemable in Littercoin. Each layer has a "Created By" stamp, which is used to display either an anonymous user (the default privacy setting of every user) or it will reveal a given name and/or username for history to remember. 


\section{Settings}

Users can visit openlittermap.com/settings to update their supplied information (name, username, email, password); delete their account; change, cancel or reactivate their subscription options; toggle the anonymity of their name and username from the default of anonymous to public; submit a public Ethereum wallet to claim Littercoin; and toggle the default presence value of the litter they are mapping.

\section{Presence}

By default, OpenLitterMap assumes that litter is remaining when and where it was recorded. The default presence value can be toggled in settings or per image during processing.

\section{Privacy and Gamification}

Users should express caution when choosing to engage in litter mapping, as it is a practice that could potentially reveal patterns about ones location and behaviour. By default, all users are anonymous and cannot compete for visibility in any of the multiple leaderboards. Similarly, the default user with anonymous settings is not credited with their given information for uploading images on the maps. To comply with GDPR (General Data Protection Regulation), several updates are being made. For example, in late April 2018, the URL to a photo was was replaced by the users' ID to a year / month / date syntax which not only gives users more privacy, but also gives OpenLitterMap improved data structuring. Currently all users' phone models are publicly visible and we will also make this anonymous by default before the launch of GDPR. Additional updates must also be made, including a restructuring and simplification of the Terms and Conditions and Privacy Policy, and giving the user the ability to download all of their data and more. Users who change the default settings of either their name and/or username to public consent to be publicly credited with given information for each image they upload on the maps. Only the users with public settings can compete for a position in any of the multiple different leaderboards that are available at each layer, simply by uploading geotagged images of litter from that location. Only users with public settings who created a location in the database will receive credit for creating that location in the database.

\section{Results and discussion}

Urban litter mapping is a new field of GIScience and OpenLitterMap offers an intricate way of mapping and producing open geospatial data on a limited but increasing variety of contemporary anthropogenic litter including corporate brands anywhere. All available data has gone through a verification process to ensure quality control before being released as free, open, and accessible for anyone to access through maps or CSV (Comma Separated Value) downloads. Although processing and verification is currently a manual process, this is being done to develop a trusted database that can train machine-learning algorithms to make manual processing less cumbersome. It is hoped that mapped and open data on plastic pollution can raise awareness about and find solutions to plastic pollution, which has been of urgent concern for decades. The maps can be used as educational tools to communicate the distribution and abundance of litter and the data can be downloaded for free and used for any purpose. Although research with OpenLitterMap data is largely unexplored, there are a large variety of pending research questions. For example, although data remains low and is prone to observer bias, experimentation with the platform could prove insightful. Do new users who may have previously littered change their behaviour after being exposed to the maps or data collection process? What spatial relationships can be identified between OpenLitterMap and OpenStreetMap data? Do different types of litter exhibit particular patterns with distance from cafés, supermarkets, bus stops or corner stores? Can this information be used to change how plastic retailers must account for the waste they facilitate through their economic activity? What influence do socio-economic characteristics, such as income, demographics or the deprivation index have on litter? Can litter data improve our measurement of the deprivation index? How effective are bin catchments at reducing littering, and could these services be more appropriately located elsewhere? Where are the high-risk areas where litter is more likely to enter the ocean? How does litter change throughout the seasons? How does city, suburban or rural litter differ by day and night, by the seasons, or by weekday and weekend? How does litter move across space and time? Has management previously identified these trends and are they informed to respond appropriately? How well does this data correlate with previous sampling efforts? These are but a few examples of the research potential provided by open litter data. By producing open data on litter we can empower the geospatial community to explore issues such as these. But to achieve that, crowdfunding, data processing, capacity building and technological developments need to be supported. New community events are needed, such as the "Global OpenLitterMapping Marathon" that anyone anywhere can participate in every Saturday from 2 to 4 pm. ${ }^{17}$

According to the EU Marine Strategy Framework Directive (MSFD), $80 \%$ of marine litter originates on land (2008/56/EC) [5]. However, in 1975 alone, it has been reported that over $161,400 \mathrm{t}$ of plastic fishing gear and synthetic packaging were dumped into the ocean by fishing fleets [4]. By 2014, it has been estimated that at least 5.25 trillion pieces of plastic were floating around in the 
upper layers of the ocean [6]. Much of the millions of tonnes marine litter floating around somewhere in the oceans has only begun to degrade, which unless removed will continue to break up and persist as smaller and smaller pieces indefinitely. As plastic fragments in the oceans, it is likely to become extremely more problematic, more difficult, and more expensive to try and remove. This warrants a call for technological, socioeconomic and collective innovation putting particular focus on the pre-marine terrestrial characteristics of plastic pollution which can be used to communicate litter with people in the areas they are most familiar with. Urgent focus needs to be put on stopping additional plastic reaching the oceans, which should prove significantly cheaper than trying to deal with it later. As well as knowing when and where to apply and maximize our cleanup efforts by physically picking up, capturing and filtering out as much plastic as possible, we need geospatial knowledge to improve our ability at stopping plastic entering landfills and the marine environment where it becomes extremely problematic. OpenLitterMap is not the only litter mapping platform out there, but it is the only one that verifies all data; makes all verified data free and open; dynamically applies a weighted hexagonal spatial grid at the city layer, which can be filtered by the day and toggled by behaviour; and rewards contributors with an original blockchain token that is generated exclusively from the production of open data. Thanks to \$1000 startup credits from Amazon Web Services, anyone can currently upload 1000 images a day. However, this is not sustainable and future of OpenLitterMap is uncertain. Despite this, OpenLitterMap could easily be sustained through a micro-financial crowdfunding campaign if society thinks this platform is important and should be maintained. Although Local Authorities could be the largest consumers of this data, they are not typically interested in challenging the status quo and revealing how polluted their cities are by the products of global corporations. Until recently, litter mapping has been taboo and not seen as being good for business. However, with the introduction of gamification and Littercoin, litter mapping could generate commercial success for supporting organisations. OpenLitterMap has not had much feedback from the geospatial community. Therefore, pending updates may become more informed through experimentation, feedback and review. The data types were carefully chosen from years of independent research and are continually being improved following newly identified items and valuable community feedback. To generate additional discussion, an independent review of all of the different litter platforms and methodologies would make a welcome addition to the litter mapping dialogue and help the geospatial community improve our efforts at tackling plastic pollution.

\section{Conclusion}

Littering costs taxpayers millions in nearly every country every year for clean up intervention and management, and trillions more in the loss of biodiversity, ecosystem services, and our general well-being, productivity, and mental health. Despite first being recognized to have a global distribution in the marine environment nearly half a century ago, the amount of plastic entering the marine environment is expected to increase exponentially if current trends continue. Cleanups and removal efforts need to remain ongoing but to improve decisions and behaviour, new insights are needed about our geospatial relationship with plastic. OpenLitterMap empowers anyone with a smartphone with the ability to generate cryptocurrency by mapping and producing open geospatial data on plastic pollution and the brands of a handful of global corporations anywhere. This platform is being developed independently by one self-taught developer and makes the mapping and provision of accurate geospatial data on plastic pollution openly available to everyone. Although the production of open data is currently tedious and only achievable through manual processing on a web-app, there are additional technological developments underway that will enable the rapid production of verified open litter data on a global scale. Machine learning might eliminate the need for manual processing and verification altogether. Native mobile apps for Android and iOS are also being developed. At the same time, OpenLitterMap is trying to define a monetary blockchain incentive to make the mapping of plastic pollution significantly more appealing. A whitepaper on Littercoin will follow this article outlining this vision in more detail. However, for significant technological progress to be achieved, and for OpenLitterMap to survive, this platform needs community support. In lieu of a grant or commercial features, crowdfunding is a realistic way of financing developments through the forthcoming nonprofit OpenLitterMap Foundation. Feedback and support from the geospatial community is particularly welcome to facilitate a conversation about how we can find and develop solutions to the plastic problem that is probably available right outside your door.

\section{Availability and requirements}

- Project Name: OpenLitterMap

- Project Homepage: openlittermap.com

- Operating System: Platform Independent

- Programming Languages: PHP, JavaScript, HTML, CSS

- Other Requirements: Only geotagged images can be submitted.

- Licence: ODbL - Open Database Licence, see openlittermap.com/terms 


\section{Endnotes}

${ }^{1}$ https://www.theoceancleanup.com/

${ }^{2}$ The author would like to thank and recognize the contribution of Mr. Amin Mobasheri for providing some extra context here.

${ }^{3}$ www.coastwatch.org/europe

${ }^{4}$ https://www.rte.ie/news/ireland/2017/1211/926540plastic-drink-bottles/

${ }^{5} \mathrm{http} / / / \mathrm{www} . e p a . i e /$ enforcement/report/seeit/

${ }^{6}$ https://litterati.org

${ }^{7}$ http://litterbase.awi.de/

${ }^{8} \mathrm{http}: / /$ www.marinedebris.engr.uga.edu/

${ }^{9}$ https://www.projectaware.org/diveagainstdebrismap

${ }^{10} \mathrm{https} / / / \mathrm{www} . c 0 a$ stalcleanupdata.org/

${ }^{11} \mathrm{https} / /$ medium.com/@littercoin/the-openlittermapfoundation-is-recruiting-unpaid-ambassadors-andinterns-e4b9a44e4757

${ }^{12} \mathrm{https} / /$ github.com/nativescript-vue/vue-cli-template ${ }^{13} \mathrm{https}$ //github.com/nativescript-vue/nativescript-vue

14 - Note, these items are being moved to a sibling closed-data platform, which is being developed at https://druglitter.info.

15* A Pathway, such as a gutter in the street, is where litter can disappear from the urban environment and may begin its journey to the ocean. OpenLitterMap gives users the ability to open up data on the location of gutters and other pathways as a means to test the theory of the "illusion of urban cleaning" whereby storm drains and gutters double up as a means to transport litter out of sight and mind (See: openlittermap.com/about).

${ }^{16}$ Note, dozens of other brands have been identified and will be added in due course.

${ }^{17}$ https://medium.com/@littercoin/open-litter-mappingmarathons-bf2cb222ab2b

\section{Abbreviations \\ AWI: Alfred Wegner Institute; AWS: Amazon Web Services; BA: Bachelor of Arts; CLI: Command Line Interface; CSV: Comma Separated Value; DAO: Decentralized Autonomous Organisation; FOSS4G: Free and Open Source Software for Geoinformatics; LTRX: Littercoin; MSFD: Marine Strategy Framework Directive; MSc: Master of Science; NOAA: National Oceanographic and Atmospheric Administration; NSW: New South Wales.; NUIG: National University of Ireland Galway.; OLM: OpenLitterMap.; OSM: OpenStreetMap.; UCC: University College Cork.; UNEP: United Nations Environment Programme.; VGI: Volunteered Geographic Information.}

\section{Acknowledgements}

The author would like to send thanks to the Editor and Reviewers for their guidance and patience in helping to improve the readability and structure of this intricate and evolving platform. In particular, the author would like to acknowledge Mr. Amin Mobasheri for proofreading and providing comments that helped with some of the finishing touches of this paper. Ultimately, OpenLitterMap would not exist without the skills and knowledge shared by the professors at the Geography Departments of University College Cork, (2009-2012 and 2013-2014) and the National University of Ireland, Galway (2014-2015). Similarly, the vision and determination for developing a Citizen Science platform from scratch would not have been inspired and nurtured without the expertize shared by the members of the COST ENERGIC Action IC1203 (vgibox.eu) who are at the forefront of advancing and promoting some of the most advanced research in Geographic Information Science. Special thanks must also be given to the inspirational work being done by all the others around the world doing exemplary work on the plastic crisis. And last but not least, none of this would have been possible without a supportive and loving family. For all of this the author is eternally grateful and would like to offer the most sincere thanks to everyone who was always there to help. OpenLitterMap is dedicated to you.

\section{Funding}

OpenLitterMap is entirely dependent on (and lacking in) crowdfunding. A non-profit, the OpenLitterMap Foundation, is being established to accept donations and give members a voice in the future of the ecosystem. Several grants are being applied for which the Foundation can help with.

\section{Author's contributions}

Seán Lynch is the author and self-taught developer of OpenLitterMap. In 2015 he achieved a first-class masters dissertation for a OGIS-based FOSS4G littermapping methodology to achieve a MSc in Coastal \& Marine Environments following a MSc in GIS \& Remote Sensing. He has developed and financed this project independently. The author read and approved the final manuscript.

\section{Author's information}

- Seán Lynch is a self-taught developer. He is the only person in his native Ireland to graduate with one MSc in GIS \& Remote Sensing and a second MSc in Coastal \& Marine Environments. Seán began researching litter in 2010 as part of his first dissertation in Walkability to achieve a BA in Geography \& Economics in University College Cork and finished his academic training with a first-class honours FOSS4G litter-mapping dissertation which contextualized and informed the fundamentals of OpenLitterMap today. After the BA he worked as a team leader for a government energy saving program in Australia; Following this he was able to finance scuba divemaster training in Thailand; On arrival home he went back to college for a Masters and was lucky enough to spend the better part of 3 -years participating with and learning from the EU COST ENERGIC Action IC1203 on Citizen Science and VGI; In 2015 he spent over 5-weeks training with 50 inspirational students and several academic experts in biological oceanography facilitated by the Alfred Wegner Institute on-board the R.V. Polarstern (PS-95) from Bremerhaven to Cape Town along the North-South Atlantic Transect. All of these have played a significant role in developing the OpenLitterMap platform and methodology. In mid-October 2017, Seán gave a talk at TEDxTallaght on OpenLitterMap that is now online. He is currently based in Ireland and would like to explore a $\mathrm{PhD}$ and further publications in litter science and has identified several innovative publications to advance our research and ability at tackling plastic pollution, which Seán believes is not only one of the most urgent and pressing issues of our time but one of the most solvable.

Linkedln: https://linkedin.com/in/seanlynchgis

TedxTallaght: https://www.youtube.com/watch?v=my7Cx-kZhT4\&t=4s

Competing interests

The author declares that he/she has no competing interests.

\section{Publisher's Note}

Springer Nature remains neutral with regard to jurisdictional claims in published maps and institutional affiliations.

Received: 25 October 2017 Accepted: 20 April 2018

Published online: 25 June 2018

\section{References}

1. Abu-Hilal, A., Al-Najiar, T., (2004)., Litter pollution on the Jordanian shores of the Gulf of Aqaba (Red Sea). Mar Environ Res Vol. 58, p.39-63. http://doi: https://doi.org/10.1016/j.marenvres.2003.12.003

2. Bakillah M, Mobasheri A, Rousell A, Hahmann S, Jokar J, Liang SH. Toward a collective tagging android application for gathering accessibility-related geospatial data in European cities. Parameters. 2014;10:21. 
3. Certoma C, Corsini F, Rizzi F. Crowdsourcing urban sustainability. Data people and technologies in participatory governance. Futures. 2015;74: 93-106.

4. Derraik JGB. The pollution of the marine environment by plastic debris: a review. Mar Pollut Bull. 2002;44:842-52. https://doi.org/10.1016/S0025326X(02)00220-5

5. Directive 2008/56/EC of the European Parliament and of the Council of 17 June 2008 establishing a framework for community action in the field of marine environmental policy: The Marine Strategy Framework Directive. Official Journal of the European Union. eur-lex.europa.eu/LexUriServ/ LexUriServ.do?uri=CELEX:32008L0056.

6. Eriksin M, Lebreton LCM, Carson HS, Thiel M, Moore CJ, Borerro JC, Galgan F, Ryan PG, Reisser J. Plastic pollution in the World's oceans: more than 5 trillion plastic pieces weighing over 250,000 tons afloat at sea. PLoS One. 2014;9(12):e111913. https://doi.org/10.1371/journal.pone.0111913

7. Goodchild MF. Citizens as sensors: the world of volunteered geography. GeoJournal. 2007:69(4):211-21.

8. Goodchild MF, Glennon JA. Crowdsourcing geographic information for disaster response: a research frontier. International Journal of Digital Earth. 2010;3(3):231-41.

9. Green ALR, Putschew A, Nehls T. Littered cigarette butts as a source of nicotine in urban waters. J Hydrol. 2014;519, p:3466-74. https://doi.org/10. 1016/j.jhydrol.2014.05.046

10. Haklay M, Weber P. Openstreetmap: User-generated street maps. IEEE Pervasive Computing. 2008;7(4):12-8.

11. Hoornweg, Daniel; Bhada-Tata, Perinaz. 2012. What a Waste: A Global Review of Solid Waste Management. Urban development series;knowledge papers no. 15. World Bank, Washington, DC. (c) World Bank. https:// openknowledge.worldbank.org/handle/10986/17388 License: CC BY 3.0 IGO.

12. International Geologic Congress., (2016)., https://www.theguardian.com/ environment/2016/aug/29/declare-anthropocene-epoch-experts-urgegeological-congress-human-impact-earth

13. Irwin A. Citizen science: a study of people, expertise, and sustainable development. London and New York: Routledge; 1995.

14. Jambeck JR, Geyer R, Wilcox C, Siegler T, Perryman M, Andrady A, Narayan R, Law KL. Plastic waste inputs from land into the ocean. Science. 2015; 347(6223):768-71. https://doi.org/10.1126/science.1260352

15. Kanhere, S. S. (2013, February). Participatory sensing: Crowdsourcing data from mobile smartphones in urban spaces. In International Conference on Distributed Computing and Internet Technology (pp. 19-26). Springer, Berlin, Heidelberg.Laurent, C.M.L., van der Zwet, J., Damsteeg, J.W., Slat, B., Andrady, A., Reisser, J., (2017)., River plastic emissions to the world's oceans., Nature., doi:https://doi.org/10.1038/ncomms15611

16. Lebreton, L., Slat, B., Ferrari, F., Sainte-Rose, B., Aitken, J., Marthouse, R., Hajbane, S., Cunsolo, S., Schwarz, A., Levivier, A., Noble, K., Deblejak, P., Maral, H., Schoeneich-Argent, R., Brambini, R., and Reisser, J., (2018)., Evidence that the great Pacific garbage patch is rapidly accumulating plastic. Nature Scientific Reports., Vol. 8, Article number 4666., doi:https://doi.org/10.1038/ s41598-018-22939-w.

17. Lebreton, L., van der Zwet, J., Damsteeg, J.W., Slat, B., Andrady, A., Reisser, J., (2017)., River plastic emissions to the world's oceans., Nature, doi:https://doi. org/10.1038/ncomms15611.

18. Mobasheri A, Deister J, Dieterich H. Wheelmap: the wheelchair accessibility crowdsourcing platform. Open geospatial data. Software and Standards. 2017;2(1):27.

19. O'Hara K, ludicello S, Bierce R. A Citizen's guide to plastics in the ocean: more than a litter problem. Washington DC: Center for Marine Conservation; 1988.

20. Pham CK, Ramirez-Llodra E, Alt CHS, Amaro T, Bergmann M, Canals M, Company JB, Davies J, Duineveld G, Galgani F, Howell KL, Huvenne VAl, Isidro E, Jones DOB, Lastras G, Morato T, Gomes-Pereira JN, Purser A, Stewart H, Tojeira H, Tubau X, Van Rooij D, Tyler PA. Marine litter distribution and density in European seas, from the shelves to deep basins. PLoS One. 2014; 9(4) https://doi.org/10.1371/journal.pone.0095839.

21. Rees, G., Pond, K., (1995)., Marine Litter Monitoring - A Review of Methods with Special Reference to NationalSurveys. Marine Pollution Bulletin. Vol. 30(2), pp. 103-108. 0025-326X(94)00192-8.

22. Rousell, A., Hahmann, S., \& Mobasheri, A. (2016, June). A two-tiered approach to OSM data collection for novice users. In Proceedings of the 19th AGILE international conference on geographic information science, Helsinki, Finland (pp. 14-17).
23. Schmidt C, Krauth T, Wagner S. Export of plastic debris by rivers into the sea. Environmental Science \& Technology. 2017; https://doi.org/10.1021/acs. est.7b02368

24. Tekman, M.B., Gutow, L., Macario, A., Haas, A., Walter, A., Bergmann, M. (2017)., Litterbase., Alfred Wegener Institute Helmholtz Centre for Polar and Marine Research., URL: litterbase.awi.de., accessed: $18^{\text {th }}$ March 2018.

25. UNEP. Marine litter: A global challenge. UNEP: Nairobi; 2009. p. 232.

26. UNEP. Valuing plastics: the business case for measuring, managing and disclosing plastic use in the consumer goods. Division of Environmental Policy Implementation: Industry; 2014

27. Zalasiewicz J, Waters CN, Summerhayes CP, Wolfe AP, Barnosky AD, Cearreta A, Cruzten P, Ellis E, Fairchild IJ, Galuszka A, Haff P, Hajdas I, Head MJ, Ivar do Sul JA, Jeandel C, Leinfelder R, McNeill JR, Neal C, Odada E, Oreskes N, Steffen W, Syvitsky J, Vidas D, Wagreich M, Williams M. The working group on the Anthropocene: summary of evidence and interim recommendations. Anthropocene. 2017;19:55-60.

\section{Submit your manuscript to a SpringerOpen ${ }^{\circ}$ journal and benefit from:}

- Convenient online submission

- Rigorous peer review

- Open access: articles freely available online

- High visibility within the field

- Retaining the copyright to your article

Submit your next manuscript at springeropen.com 